\title{
¿Por qué la mayoría de la investigación clínica no es útil?
}

\author{
Why most clinical research is not useful?
}

\begin{abstract}
Resumen
En este ensayo, el autor reflexiona sobre la relevancia de las investigaciones clínicas que se desarrollan con frecuencia en la actualidad. Destaca que a diferencia de la "investigación creativa, especulativa o basada en la curiosidad" (blue-sky research), que no puede ser juzgada directamente en base al impacto práctico, la investigación clínica debería ser útil: es decir, hacer una diferencia para la salud de los pacientes, o ser realizada con una perspectiva realista. Para ello realiza algunas propuestas de mejora, como ser; una correcta justificación y puesta en contexto del problema en estudio, procurar una adecuada ganancia de información, pragmatismo, estar centrada en los pacientes, ser viable, con una adecuada relación precio-calidad y transparente. Muchos estudios, aún en la mayoría de las revistas médicas generales, no satisfacen ninguna de estas características, y muy pocos satisfacen la mayoría o todas ellas. Gran parte de la investigación falla en su utilidad no por sus hallazgos, sino por su diseño. Por otro lado, el autor sostiene que las fuerzas que guían la producción y diseminación de investigación clínica inútil son claramente identificables y modificables. Se necesita una reforma. Alterar nuestro abordaje podría producir fácilmente más investigación clínica útil y, al mismo tiempo, de menor costo.
\end{abstract}

\section{Abstract}

In this essay, the author reflects on the relevance of today's clinical research. Stresses that unlike blue-sky research, which can not be judged directly based on practical impact, clinical research should be useful: that is, make a difference to the health of patients, or be carried out with a realistic perspective. Proposed key features of clinical investigation to achieve that are: problem based, context placement, information gain, pragmatism, patient centered, value for money, feasibility and transparency. Many studies, even those published in core clinical journals, do not satisfy any of these characteristics, and very few satisfy most or all of them. Most research fails in its utility not because of its findings, but because of its design. On the other hand, the author argues that the forces that guide the production and dissemination of useless clinical research are clearly identifiable and modifiable. A reform is needed. Altering our approach could easily produce more useful clinical research and, at the same time, with lower cost.

Traducido y adaptado de: Ioannidis JPA. Why Most Clinical Research Is Not Useful. PLoS Med. 2016;13(6): e1002049. doi:10.1371/journal.pmed.1002049. Con autorización del autor y la empresa editorial bajo el reglamento de Creative Commons Attribution Licence (Open Access).

\section{Introducción}

Los médicos asistenciales y otros profesionales de la salud estarán familiarizados con lo poco útil que resultan las pulicaciones que se pueden encontrar en las revistas médicas. El término "investigación clínica" intenta abarcar todos los tipos de investigación que encaran preguntas sobre el tratamiento, la prevención, el diagnóstico, el rastreo o el pronóstico de las enfermedades; o el mejoramiento y el mantenimiento de la salud. Los estudios sobre intervenciones experimentales (ensayos clínicos) constituyen el mejor diseño para responder estas preguntas; sin embargo, muchos estudios observacionales también pueden ofrecer evidencia relevante.

Que la investigación clínica sea "útil" significa que esta pueda promover un cambio favorable en la toma de decisiones (en cuanto a beneficios, daños, costos y cualquier otro impacto considerado) ya sea por sí misma o cuando se integra con otros estudios y evidencias mediante revisiones sistemáticas, meta-análisis, análisis de decisión y guías de práctica clínica.

Existen millones de artículos científicos (se han publicado aproxi- madamente un millón de ensayos clínicos, junto a decenas de miles de revisiones sistemáticas) pero la mayoría de ellos no son útiles. Se estima que el desperdicio de la investigación médica (clínica o de otros tipos) consume hasta el $85 \%$ de los miles de millones gastados cada año ${ }^{1}$. El autor ha escrito anteriormente acerca de por qué la mayor parte de la investigación publicada es falsa ${ }^{2}$ y cómo hacer para que esta resulte más confiable ${ }^{3}$. Para que sea útil, la investigación clínica tiene que ser verídica, pero esto por si solo no es suficiente. En este artículo se describen las características claves de la investigación clínica útil (ver Tabla 1) y el estado actual, y se sugieren posibilidades de mejora para el futuro.

Transformar la investigación creativa, especulativa o basada en la curiosidad (blue-sky research) en un proceso más productivo representa un problema intrincado, dado la poca predictibilidad de este tipo de investigación. Sin embargo, pensar en procesos de mejora significativos de la investigación clínica y el desarrollo de herramientas que ayuden a determinar su utilidad o futilidad, parecen conceptualmente más claros.

Tabla 1. Características a considerar a la hora de evaluar si una investigación clínica es útil.

\begin{tabular}{|l|l|}
\hline Característica & ¿Existe un problema importante o significativo que necesite solución? \\
\hline Justificación & ¿Se ha evaluado de manera sistemática la evidencia ya disponile para identificar la necesidad de nuevos estudios? \\
\hline Puesta en contexto & $\begin{array}{l}\text { ¿El estudio de investigación propuesto, es lo necesariamente grande y extendido en el tiempo como para brindar } \\
\text { información suficiente? }\end{array}$ \\
\hline Ganancia de información & ¿La investigación refleja los acontecimientos de la vida real? ¿Importa si se desvía? \\
\hline Pragmatismo & ¿La investigación refleja las prioridades más importantes de los pacientes? \\
\hline Investigación centrada en el paciente & ¿Esta investigación justifica la inversión monetaria? \\
\hline Relación precio-calidad & ¿Esta investigación puede realizarse? \\
\hline Trabilidad & ¿Son los métodos, datos y análisis verificables y carentes de sesgos? \\
\hline
\end{tabular}




\section{Características de la investigación clínicamente útil}

\section{Justificación}

Resulta claramente útil resolver aquellos problemas relacionados con una alta carga de enfermedad. Sin embargo, el contexto también es importante. La resolución de problemas de baja prevalencia pero con consecuencias serias para los pacientes afectados también resulta valioso. A su vez, de estos problemas podrían surgir investigaciones con aplicabilidad extendida si el conocimiento resulta relevante a afecciones comunes. Por ejemplo, descubrir la importancia de la pro-proteína-convertasa subtilisina-kexina tipo 9 (abreviadamente PCSK9) en el patrón de la hipercolesterolemia familiar, permitiría desarrollar tratamientos para muchos pacientes con enfermedad cardiovascular aunque sin ese patrón. Además, en el caso de epidemias explosivas (por ejemplo, de ébola), se podría asimismo considerar la carga potencial carga de enfermedad si la epidemia saliese de control.

Por el contrario, la investigación clínica se torna realmente fútil cuando se crea una percepción ficticia de carga de enfermedad entre la gente sana ("disease mongering"). En esas circunstancias, las personas tratadas no se benefician, simplemente porque no existe una verdadera enfermedad.

Los datos muestran sólo una correlación modesta entre el número de investigaciones y la carga de diversas enfermedades ${ }^{5,6}$. Además, el "disease mongering" afecta a múltiples especialidades médicas ${ }^{4,7,8}$.

\section{Puesta en contexto y ganancia de información}

La investigación clínica útil procura la adquisición de información clínicamente relevante ${ }^{9}$, es decir, agregar valor a lo que ya sabemos. Esto significa que, en primer lugar, necesitamos repasar lo que ya conocemos para que la nueva información pueda ser puesta en contexto $^{10}$. En segundo lugar, los estudios deberían ser diseñados de manera tal que provean información suficiente como para asegurar que tanto los pacientes, los médicos y los agentes de toma de decisiones sanitarias puedan tener confianza acerca de la magnitud y la índole de los beneficios y los daños de una determinada práctica. Estos estudios deberían ser juzgados en base a su impacto clínico y su capacidad para cambiar la práctica. Idealmente, los estudios que se inician deberían ser clínicamente útiles, independientemente de sus resultados. Si la utilidad de un estudio esta condicionada en función de si alcanza o no un determinado resultado, podría generarse cierta presión para obtenerlo o para interpretar la información como si ese resultado se hubiera obtenido.

La mayoría de las investigaciones no están precedidas de una revisión sistemática sobre el tema ${ }^{10,11}$. Con frecuencia las intervenciones terapéuticas se comparan contra placebo o contra el cuidado habitual, a pesar de existir otras intervenciones con efectividad ya demostrada. Los cálculos del tamaño muestral habitualmente evalúan a cada ensayo clínico de manera individual, ignorando otros estudios relacionados. En todo PubMed, la mediana de tamaño muestral para ensayos clínicos aleatorizados en 2006 fue de 36 por cada rama de tratamiento ${ }^{12}$. Además, es habitual que se utilicen, en pos de obtener un adecuado poder estadístico, resultados subrogantes (intermedios), no validados y carentes de sentido clínico ${ }^{13}$, así como resultados compuestos que combinan resultados de diferente relevancia clínica ${ }^{14}$. Por último, al diseñar los estudios clínicos, rara vez se discute el valor de los resultados "negativos".

\section{Pragmatismo}

Las inferencias de las investigaciones deben ser aplicables a las circunstancias de la vida real. Cuando el contexto de los estudios de investigación clínica se desvía de las circunstancias típicas de la vida real, la pregunta que los lectores críticos deben hacerse es: ¿hasta qué punto estas diferencias invalidan las principales conclusiones del estudio?
Una concepción habitualmente errónea, respecto de la generalización de los resultados de los estudios, es que la muestra de un ensayo clínico debe ser totalmente representativa de la población general de pacientes (en el caso de preguntas de tratamiento), o de la comunidad entera (en el caso de preguntas de prevención). Debido a que los ensayos clínicos dependen del consentimiento de los participantes, ningún ensayo clínico contiene un muestra aleatoria perfecta de la población general. Sin embargo, cuando se utilizan diseños pragmáticos es posible capturar las circunstancias de la vida real y los efectos del tratamiento de una manera que pueden resultar similares a las de los individuos no participantes, independientemente de la representatividad de la muestra del estudio.

Si bien desde hace tiempo se promueve el pragmatismo en el ámbito de la investigación ${ }^{15}$, resulta raro encontrarlo. Entre 1996 y 2010, de acuerdo a una revisión sistemática, solamente se publicaron nueve ensayos clínicos pragmáticos de efectividad comparada de drogas con financiamiento de la industria biomédica ${ }^{16}$, mientras que se publicaron miles de ensayos clínicos de eficacia explorando la optimización de las circunstancias experimentales.

Si bien resulta atractivo el estudio de los efectos del tratamiento de los ensayos clínicos bajo condiciones "ideales", quedan dudas acercas de la generalizabilidad de los hallazgos en las circunstancias de vida real. Por su parte, se considera en general pobre la capacidad de los estudios observacionales (realizados de a miles) para responder preguntas acerca del efecto causal de los tratamientos ${ }^{17}$. El uso de la información recolectada de manera rutinaria se publicita como más representativo de la vida real, aunque esto con frecuencia no es cierto. La mayoría de los estudios observacionales lidian con poblaciones particulares (por ejemplo, enfermeras, médicos o trabajadores) y/o circunstancias peculiares (por ejemplo, pacientes atendidos en sistemas de salud especializados o cubiertos por un tipo de seguro en particular, o con determinados criterios de inclusión para un registro). Eventualmente, los estudios observacionales a menudo sobreestiman sustancialmente los efectos de los tratamientos ${ }^{18,19}$.

\section{Centrada en el paciente}

La investigación útil es aquella centrada en el paciente ${ }^{20}$. Se realiza para beneficiar a los pacientes o preservar su salud y mejorar su bienestar, y no para cumplir las necesidades de los médicos, investigadores o patrocinantes. La investigación clínica útil debe estar alineada con las prioridades de los pacientes, las utilidades que los pacientes asignan a los diferentes problemas y resultados, y con la aceptabilidad que ellos le asignan a las intervenciones durante el periodo en el cual están indicadas. Los resultados subrogantes propuestos en las investigaciones deben estar estrechamente correlacionados con los resultados clínicos verdaderamente relevantes para los pacientes.

Actualmente hay un interés renovado en la investigación centrada en los pacientes, ejemplificado en el Instituto de Investigación de Resultados Centrados en los Pacientes (PCORI, por sus iniciales en inglés), que fue lanzado en 2012 en los EE.UU. para fomentar la investigación relevante a las necesidades de los pacientes ${ }^{21}$. Además se están desarrollando actividades similares en el Reino Unido y otros lugares. Sin embargo, en raras ocasiones se involucra a los pacientes en la determinación de las prioridades de investigación, y es frecuente que las prioridades de los pacientes y las agendas de investigación sean discrepantes. Los pacientes y los médicos se encuentran bombardeados con información que trata de convencerlos de que los resultados subrogantes o irrelevantes son importantes. Tales atajos suelen tener beneficios económicos, acerelar la publicación de estudios y la promoción académica.

\section{Relación precio-calidad}

La relación precio-calidad es una consideración importante, especialmente en una era en que los recursos son limitados, y esto puede 
ser evaluado con un modelo formal ("valor de la información") 22. Los diferentes estudios pueden requerir diferentes niveles de inversión financiera, y a su vez, pueden diferir sustancialmente en cuánto podemos aprender de ellos. Sin embargo, los beneficios de la investigación clínica útil sobrepasan los costos que insume realizarla ${ }^{23}$. La mayoría de los métodos para calcular la relación precio-calidad siguen siendo constructos teóricos, y las aplicaciones prácticas de los métodos de valor por información se cuentan con los dedos de la mano ${ }^{24}, 25$. La investigación clínica sigue siendo extremadamente cara, aunque se estima que se podría eliminar el $90 \%$ de los costos de los ensayos clínicos actuales ${ }^{26,27}$. La reducción de los costos, haciendo más eficiente la investigación, podría dejar recursos para investigar más. Sin embargo, la reducción en la presión por realizar recortes presupuestarios, que conduce a estudios con insuficientes poder, precisión y duración, además de resultados inadecuados, podría también ayudar a mejorar la calidad de la investigación y a cambiar convincentemente la práctica clínica.

\section{Viabilidad}

Aun cuando todas las demás características se cumplan, algunos estudios pueden ser muy difíciles o prácticamente imposibles de realizar. La viabilidad de la investigación a veces puede ser difícil de predecir de antemano, y puede haber un optimismo injustificado entre los investigadores y los financiadores.

Muchos ensayos clínicos finalizan por futilidad. Entre 2000 y 2003 se discontinuaron el $25 \%$ de los ensayos clínicos aprobados por seis comités de ética en Canadá, Alemania y Suiza ${ }^{28}$, mientras que la tasa de discontinuación fue de $43 \%$ para una cohorte de ensayos clínicos de intervenciones quirúrgicos registrados entre 2008 y $2009^{29}$. Para otros tipos de investigación los problemas de viabilidad se conocen menos, aunque probablemente sean aún más comunes.

\section{Transparencia (Confianza)}

La utilidad disminuye cuando la investigación no es transparente, cuando los datos de los estudios, sus protocolos y otros procesos no están disponibles para la verificación o el uso ulterior por parte de terceros. La confianza también se erosiona cuando hay grandes sesgos en el diseño, la conducción y el reporte de la investigación. Sólo el $61 \%$ de los ensayos clínicos publicados en 2010 habían sido registrados ${ }^{30}$, siendo las tasas de registro mucho más bajas para las intervenciones no reguladas ${ }^{31}$ (por ejemplo, $21 \%$ y $29 \%$ de los ensayos clínicos publicados en revistas de terapias psicológicas o comportamentales $^{32}$ y aquellos publicados en revistas de terapias físicas $^{33}$, respectivamente). Solamente el 28\% (55/200) de las revistas que publican ensayos clínicos exigían el registro del estudio durante $2012^{34}$. Se registran pocos protocolos completos, casi nunca se pre-especifican los planes de análisis, y raramente están disponibles los datos de los estudios completos ${ }^{35}$. La confianza se erosiona cada vez que, ya sea mediante procedimientos legale ${ }^{36} \mathrm{o}$ por la publicación de reanálisis ${ }^{37}$, se descubre una gran subversión de la evidencia con conclusiones diferentes (por ejemplo, como en el caso de los inhibidores de la neuraminidasa para el tratamiento de la influenza) ${ }^{38}$. Además, los sesgos en el diseño, análisis, reporte e interpretación son muy prevalentes ${ }^{39-41}$.

\section{Otras consideraciones}

Incertidumbre. Puede existir cierta incertidumbre en cada una de las características de la investigación clínica delineadas previamente, aun cuando habitualmente sea menor que la incertidumbre inherente a la investigación creativa (blue-sky research) y la investigación preclínica. La incertidumbre también evoluciona a través del tiempo, especialmente cuando el esfuerzo puesto en la investigación se prolonga por años. Los interrogantes también pueden perder su importancia cuando cambia el contexto. En uno de los primeros artículos escritos por el autor, una revisión sistemática de monoterapia con zidovudina $^{42}$, la pregunta era extremadamente relevante al comenzar la investigación en 1993, y todavía lo era cuando el artículo fue aceptado a fines de 1994. Sin embargo, para el momento en el artículo fue publicado, a mediados de 1995, ya no tenía valor, dada la emergencia de nuevos tratamientos de alta eficacia: la utilidad clínica fue demolida por los avances tecnológicos.

Otras fuentes de evidencia además de los ensayos clínicos. Los estudios observacionales a menudo agregan más confusión que resolución a los déficits de información ${ }^{18,19}$. Por su parte, los metaanálisis, análisis de decisión y guías de práctica clínica no logran aclarar el panorama cuando se fundamentan en estudios inútiles, y a su vez, pueden agregar sus propios problemas y sesgos ${ }^{43-45}$.

Enfocándose en revistas importantes. Algunos médicos prefieren leer las investigaciones publicadas sólo en las revistas médicas más importantes (p.ej. The New England Journal of Medicine, The Lancet, BMJ, JAMA, y PLOS Medicine). Sin embargo, estas revistas abarcan una pequeña minoría de los estudios de investigación publicados. De los 730.447 artículos etiquetados como "ensayos clínicos" en PubMed al 26 de Mayo de 2016, solo 18.231 estaban publicados en las revistas más importantes. La mayoría de los artículos incluídos en las guías de práctica clínica se publican en otros lugares. Los

Tabla 2. ¿Cuán a menudo se cumplimentan las características de utilidad en los estudios publicados en las revistas importantes y en toda la investigación clínica?

\begin{tabular}{|l|l|l|}
\hline Característica de utilidad & Estudios publicados en revistas importantes & \multicolumn{2}{l|}{ Toda la investigación clínica } \\
\hline Justificación & Variable & Minoría \\
\hline Puesta en contexto & Variable por revista (infrecuente) & Infrecuente \\
\hline Ganancia de información & Mayoría & Raras veces \\
\hline Pragmatismo & Raras veces & Raras veces \\
\hline Investigación centrada en el paciente & Raras veces/Infrecuente & Raras veces \\
\hline Relación precio-calidad & No se sabe/Raras veces & No se sabe/Raras veces \\
\hline Viabilidad & Casi siempre & Mayoría \\
\hline Transparencia & $\begin{array}{l}\text { Raro/Infrecuente (datos compartidos)* } \\
\text { Casi siempre (registro de ensayo clínico) }\end{array}$ & $\begin{array}{l}\text { Raro/Infrecuente, excepto para registro de } \\
\text { ensayos clínicos (aun así, sólo una minoría) }\end{array}$ \\
\hline
\end{tabular}

a Rara vez: en <1\% de los estudios; infrecuente (1 a 20\%); minoría (20 a 50\%); mayoría (50 a 80\%); muy común ( 80 a $99 \%$ ); casi siempre (>99\%). ( ${ }^{*}$ ) La situación está mejorando en los últimos años para los ensayos clínicos. 
estudios publicados en las revistas más importantes pueden ser mejores en el sentido de abarcar problemas importantes, pero dada su visibilidad, también pueden propagar más "disease mongering" que otras revistas menos visibles. Los ensayos clínicos publicados en revistas importantes son, en promedio, de mayor tamaño (p. ej. una mediana de tamaño muestral de 3.116 y 3.104 para artículos publicados en The Lancet y BMJ, respectivamente, hasta septiembre de $2007^{46}$ ). Sin embargo, los ensayos clínicos pequeños publicados en las revistas importantes tienen, en promedio, resultados más exagerados, que los estudios igualmente pequeños pero publicados en otras revistas ${ }^{47}$. The Lancet solicita de manera rutinaria y sistemática la puesta en contexto para los ensayos clínicos, y, con frecuencia, las revistas importantes solicitan los protocolos completos para los ensayos clínicos publicados. Otras características como el pragmatismo, la investigación centrada en el paciente, evaluar la relación precio-calidad, la transparencia y la protección frente al sesgo, siguen siendo subóptimas para la mayoría de los ensayos clínicos publicados en las revistas importantes (ver Tabla 2).

\section{El panorama general}

Finalmente, ninguna característica se cumple para la mayoría de los estudios de investigación clínica, quizás con la excepción de la viabilidad (ver Tabla 2). Los estudios que cumplen todas o casi todas las características de utilidad son rarezas extremas, aún en revistas altamente selectivas (ver Tabla 2).

\section{La oportunidad de mejorar la situación}

No se debería ver el problema de la investigación inútil como culpa de un grupo específico (por ejemplo, de los investigadores clínicos), sino como una oportunidad de mejora. Los retos y los problemas por resolver involucran no solo a los investigadores, sino también a las instituciones, los mecanismos de financiamiento, la industria, las revistas y muchos otros actores interesados, incluyendo a los pacientes y el público. Los esfuerzos conjuntos de los múltiples interesados pueden dar soluciones que probablemente sean adoptadas de manera más amplia y, en consecuencia, más exitosa ${ }^{3}$.

\section{La mano de obra en la investigación clínica y los médicos}

La mano de obra en la investigación clínica es enorme: millones de personas han sido coautores de al menos un artículo biomédico, y la mayoría lo ha hecho una sola vez ${ }^{48}$. Habitualmente se espera algun tipo de producción en investigación por parte de los estudiantes, los residentes y los becarios clínicos. Si bien esta exposición puede ser interesante, habitualmente se los juzga por su habilidad publicar rápidamente, un criterio desfavorable para la generación de estudios grandes, a largo plazo y realizados en equipo, que son los necesarios con frecuencia para informarnos acerca de la salud, la enfermedad y los cuidados en salud. Estos investigadores pueden ser explotados como personal voluntario o mal remunerado ${ }^{49}$. Como es de esperar, un personal poco entrenado y no comprometido, no puede producir investigación de alta calidad. Otras recetas perversas en la investigación clínica incluyen la demanda a las universidades y a otras instituciones de generar más artículos (o al menos, unidades publicables) en vez de artículos clínicamente útiles y de impacto clínico, características que no forman parte formal de la métrica de la publicación, tan a menudo usada para juzgar el desempeño académico.

Por otro lado, en vez de intentar que cada médico sea un prolíico investigador, el entrenamiento de los médicos en entender los métodos de investigación y la medicina basada en la evidencia puede ayudar a mejorar la situación, inculcando un escepticismo saludable y habilidades de pensamiento crítico.

\section{Bipolo industria-regulador y socios académicos}

$\mathrm{La}$ industria y las agencias reguladoras constituyen un bipolo estrechamente conectado en el licenciamiento de drogas y de otros productos biomédicos. La industria responde a los requerimientos regulatorios, y las agencias regulatorias actúan tanto como guardianes del bien común como facilitadores de la industria, lo que genera una tensión y una ambigüedad en su misión. La industria debería permitirse abogar por la investigación clínica útil, con reguladores que realicen un apareamiento de los beneficios comerciales con la utilidad de los productos de la industria, logrando así ayudar a que las buenas compañías superen a las malas y alineando los intereses de los accionistas con los de los pacientes y el público. Quizá sea necesario que las agencias regulatorias asuman un rol más enérgico para garantizar la conducción de mega-ensayos clínicos realmente útiles.

El financiamiento de la investigación actual incentiva la realización de estudios de corta duración que pueden ser desarrollados rápidamente para generar un resultado expeditivamente publicable, mientras que la respuesta a algunas preguntas importantes pueden requerir estudios a largo plazo, con mayores necesidades financieras que las previstas por los ciclos de financiamiento actualmente disponibles. La asociación de iniciativas de investigación centradas en el paciente ${ }^{50}$ con los "académicos" puede resolver potencialmente algunos de los retos en el diseño e implementación de ensayos clínicos más pragmáticos ${ }^{51}$. Se debería reconocer que, aún para los ensayos clínicos más eficientes, el costo puede ser sustancial si muchos de estos ensayos clínicos requieren financiamiento con fondos públicos. La industria aún podría participar mediante la contribución de recursos destinados a un fondo común, bajo control público, para ensayos clínicos conducidos por investigadores académicos sin conflicto de intereses. Un amplio financiamiento de investigación clínica útil podría estar financiado por el 1 a $2 \%$ por ciento de lo recaudado en las ventas de drogas populares ${ }^{52}$.

Agenda de financiamiento de investigación creativa, preclínica y ciencia clínica

La investigación creativa sin productos finales pre-especificados (blue-sky science) es importante y requiere del apoyo público. Sin embargo, mucha de la investigación "básica" sí tiene productos finales pre-especificados, como la investigación en el desarrollo de nuevos fármacos o pruebas diagnósticas. Esta investigación podría ser financiada por la industria o por terceros que pudieran obtener ganancias si se llegara a un producto efectivo. De esta forma, el financiamiento público que actualmente se destina a este tipo de investigación podría migrar hacia la investigación clínica útil, especialmente en muchos casos de investigaciones que no son financiados por la industria debido a ausencia de derechos de patente, y que sin embargo podrían ser útiles para mejorar el cuidado de la salud. La reasignación de fondos podría ayudar a mejorar toda la investigación (básica, preclínica y clínica, ver Tabla 3). 
Tabla 3. Financiamiento de los distintos tipos de investigación: metas pre-especificadas, utilidad, financiadores actuales y financiadores ideales.

\begin{tabular}{|l|l|l|l|}
\hline \multicolumn{2}{|l|}{ Tipo de investigación } & Producto/ meta pre-especificado & \multicolumn{3}{|c|}{ Financiadores actuales } \\
\hline Ciencia creativa (blue-sky) & Ninguno (alta incertidumbre por defecto) & Público (por ejemplo, NIH) & Público (por ejemplo, NIH) \\
\hline Investigación pre clínica aplicada & Sí (hay incertidumbre, pero debería haber metas) & Eúblico (por ejemplo, NIH) & $\begin{array}{l}\text { Emprendedores e industria, quienes } \\
\text { se beneficiarían de los productos } \\
\text { quealmente funcionan; el } \\
\text { financiamiento actual debería } \\
\text { migrar a investigación clínica }\end{array}$ \\
\hline Investigación clínica & $\begin{array}{l}\text { Sí (la incertidumbre debería ser manejada, } \\
\text { debería haber metas explícitas) }\end{array}$ & $\begin{array}{l}\text { Público (NIH, PCORI); la industria } \\
\text { podría contribuir con algunos } \\
\text { recursos destinados a un fondo } \\
\text { común; las agencias regulatorias y } \\
\text { las universidades/ institutos de } \\
\text { investigación deberían salvaguardar } \\
\text { la independencia de la investigación } \\
\text { y podrían guiar la agenda global. }\end{array}$ \\
\hline
\end{tabular}

$\mathrm{NIH}$ : National Institutes of Health; PCORI: Patient-Centered Outcomes Research Institute

\section{Revistas}

Las revistas pueden ser una importante influencia en la definición de estándares de investigación aceptable. Sin embargo organismos externos a éstas bien podrían evaluar la utilidad clínica de los artículos publicados. Por ejemplo, uno de estos organismos podría encargarse de monitorizar un "índicador de utilidad clínica de revistas" que abarque algunas de las características mencionadas previamente.

\section{Pacientes y grupos de defensoría}

Los pacientes y los grupos de defensoría son los que más se beneficiarían de un incremento en las investigaciones clínicas útiles. Estos grupos pueden ejercer una influencia positiva siempre y cuando dispongan de la destrezas técnicas acerca de cómo "se hace ciencia" y a su vez estén protegidos de influencias sesgadas. Los medios de comunicación y los comunicadores especializados en salud ${ }^{53}$ también podrían colaborar enfocándose en la necesidad de obtener investigación clínicamente útil y no conformarse con menos.

\section{Conclusiones}

En general, no sólo la mayoría de investigación es falsa, sino que además, la mayoría de los hallazgos en investigación no son útiles. Las intervenciones médicas deberían y podrían resultar en un enorme beneficio para los seres humanos. No tiene sentido realizar investigaciones clínicas sin asegurar su utilidad. Las reformas y mejoras en este aspecto vienen demoradas.

Traducido por Juan Victor Ariel Franco. Servicio de Medicina Familiar Comunitaria del Hospital Italiano de Buenos Aires. juan.franco@hospitalitaliano.org.ar

¿Por qué la mayoría de la investigación clínica no es útil? Evid Act Pract Ambul. 2018;21(1):14-19. Traducido y adaptado de: loannidis JPA. Why Most Clinical Research Is Not Useful. PLoS Med. 2016;13(6): e1002049. doi:10.1371/journal.pmed.1002049. PMID: 27328301.

\section{Referencias bibliográficas}

1. Macleod MR, y col. Biomedical research: increasing value, reducing waste. Lancet. 2014;383(9912):101-4. doi: 10.1016/S0140-6736(13)62329-6. PMID:24411643.

2. loannidis JP. Why most published research findings are false. PLoS Med. 2005;2(8):e124. PMID:16060722 doi: 10.1371/journal.pmed.0020124.

3. loannidis JP. How to make more published research true. PLoS Med. 2014;11(10):e1001747. doi: 10.1371/journal.pmed.1001747. PMID:25334033

4. Moynihan R, y col. Disease mongering is now part of the global health debate. PLoS Med. 2008;5(5):e106. doi: 10.1371/journal.pmed.0050106. PMID:18507498.

5. Evans JA, y col. Attention to local health burden and the global disparity of health research. PLoS ONE. 2014;9(4):e90147. doi: 10.1371/journal.pone.0090147. eCollection 2014. PMID:24691431.

6. Viergever RF, y col. Use of data from registered clinical trials to identify gaps in health research and development. Bull World Health Organ. 2013;91(6):416-425C. doi: 10.2471/ BLT.12.114454. PMID:24052678.

7. Moynihan R, y col. Selling sickness: the pharmaceutical industry and disease mongering. BMJ 2002;324(7342):886-91. PMID:11950740 doi: 10.1136/bmj.324.7342.886 8. Frances A. Saving normal: an insider's revolt against out-of-control psychiatric diagnosis, DSM-5, big pharma, and the medicalization of ordinary life. HarperCollins, New York, 2013 9. Evangelou E, y col. Perceived information gain from randomized trials correlates with publication in high-impact factor journals. J Clin Epidemiol. 2012; 65(12):1274-81. doi: 10.1016/J. 9. Evangelou E, y col. Perceived inform

jclinepi.2012.06.009. PMID:22959593.
10. Clarke M, y col. Discussion sections in reports of controlled trials published in general medical journals: islands in search of continents? JAMA. 1998;280(3):280-2. PMID:9676682 doi: 10.1001/jama.280.3.280.

11. Clarke M, y col. Reports of clinical trials should begin and end with up-to-date systematic reviews of other relevant evidence: a status report. J R Soc Med. 2007;100(4):187-90. PMID:17404342 doi: 10.1258/jrsm.100.4.187.

12. Hopewell S, y col. The quality of reports of randomised trials in 2000 and 2006: comparative study of articles indexed in PubMed. BMJ. 2010;340:c723. doi: 10.1136/bmj.c723. PMID:20332510.

13. Fleming TR, y col. Surrogate end points in clinical trials: are we being misled? Ann Intern Med. 1996;125(7):605-13. PMID:8815760 doi: 10.7326/0003-4819-125-7-199610010-00011. 14. Ferreira-González I, y col. Problems with use of composite end points in cardiovascular trials: systematic review of randomised controlled trials. BMJ. 2007;334(7597):786. PMID:17403713 doi: 10.1136/bmj.39136.682083.ae.

15. Tunis SR, y col. Practical clinical trials: increasing the value of clinical research for decision making in clinical and health policy. JAMA. 2003;290(12):1624-32. PMID:14506122 doi: 10.1001/jama.290.12.1624.

16. Buesching DP, y col. The role of private industry in pragmatic comparative effectiveness trials. J Comp Eff Res. 2012;1(2):147-56. doi: 10.2217/cer.12.9. PMID:24237375.

17. Prasad V, y col. Observational studies often make clinical practice recommendations: an empirical evaluation of authors' attitudes. J Clin Epidemiol. 2013;66(4):361-366.e4. doi: 10.1016/.jclinepi.2012.11.005. PMID:23384591.

10.1016/j.jclinepi.2012.11.005. PMID:23384591.
18. Hemkens LG, y col. Routinely collected data and comparative effectiveness evidence: promises and limitations. CMAJ. 2016 May 17;188(8):E158-64. doi: 10.1503/cmaj.150653.

18. Hemkens LG, y col. Routinely collected data and comparative effectiveness evidence: promises and limitations. CMAJ. 2016 May 17;188(8):E158-64. doi: 10.1503/cmaj.150653.
19. Hemkens LG, y col. Agreement of treatment effects for mortality from routinely collected data and subsequent randomized trials: meta-epidemiological survey. BMJ. $2016 ; 352: 1493$. doi: 10.1136/bmj.i493. PMID:26858277.

20. Mullins CD, y col. Patient-centeredness in the design of clinical trials. Value Health. 2014;17(4):471-5. doi: 10.1016/j.jval.2014.02.012. PMID:24969009

21. Selby JV, y col. PCORI at 3 years-progress, lessons, and plans. N Engl J Med. 2014;370(7):592-5. doi: 10.1056/NEJMp1313061. PMID:24521104.

22. Meltzer DO, y col. Minimal modeling approaches to value of information analysis for health research. Med Decis Making. 2011;31(6):E1-E22. doi: 10.1177/0272989X11412975. PMID:21712493. 
23. Detsky AS. Are clinical trials a cost-effective investment? JAMA 1989;262(13);1795-1800. PMID:2506366 doi: 10.1001/jama.262.13.1795.

24. Soares MO, y col. An evaluation of the feasibility, cost and value of information of a multicentre randomised controlled trial of intravenous immunoglobulin for sepsis (severe sepsis and septic shock): incorporating a systematic review, meta-analysis and value of information analysis. Health Technol Assess. 2012;16(7):1-186. doi: 10.3310/hta16070. PMID:22361003. 25. Reith C, y col. Randomized clinical trials-removing unnecessary obstacles. N Engl J Med. 2013;369(11):1061-5. doi: 10.1056/NEJMsb1300760. PMID:24024845.

26. Minelli $\mathrm{C}$, y col. Value of information: a tool to improve research prioritization and reduce waste. PLoS Med. 2015;12(9):e1001882. doi: 10.1371/journal.pmed.1001882. PMID:26418866. 27. Eisenstein EL, y col. Sensible approaches for reducing clinical trial costs. Clin Trials. 2008:5(1):75-84. doi: 10.1177/1740774507087551. PMID:18283084.

28. Kasenda B, y col. Prevalence, characteristics, and publication of discontinued randomized trials. JAMA. 2014;311(10):1045-51. doi: 10.1001/jama.2014.1361. PMID:24618966.

29. Chapman SJ, y col. Discontinuation and non-publication of surgical randomised controlled trials: observational study. BMJ. 2014;349:g6870. doi: 10.1136/bmj.g6870. PMID:25491195.

30. Van de Wetering FT, y col. Trial registration numbers are underreported in biomedical publications. PLoS ONE. 2012;7:e49599. doi: 10.1371/journal.pone.0049599. PMID:23166724. 31. Dal-Ré R, y col. Call to improve transparency of trials of non-regulated interventions. BMJ. 2015;350:h1323. doi: 10.1136/bmj.h1323. PMID:25820265.

32. Milette $\mathrm{K}, \mathrm{y}$ col. Transparency of outcome reporting and trial registration of randomized controlled trials in top psychosomatic and behavioral health journals: a systematic review. $\mathrm{J}$ Psychosom Res. 2011;70:205-17. doi: 10.1016/j.jpsychores.2010.09.015. PMID:21334491.

33. Babu AS, y col. Clinical trial registration in physical therapy journals: a cross-sectional study. Phys Ther. 2014:94:83-90. doi: 10.2522/pti.20120531. PMID:24009345.

34. Wager E, y col. "Hardly worth the effort"? Medical journals' policies and their editors' and publishers' views on trial registration and publication bias: quantitative and qualitative study. BMJ 2013;347:f5248. doi: 10.1136/bmj.f5248. PMID:24014339.

35. Doshi P, y col. Raw data from clinical trials: within reach? Trends Pharmacol Sci. 2013 Dec;34(12):645-7. doi: 10.1016/.tips.2013.10.006. PMID:24295825.

36. Vedula SS, y col. Outcome reporting in industry-sponsored trials of gabapentin for off-label use. N Engl J Med. 2009;361(20):1963-71. doi: 10.1056/NEJMsa0906126. PMID:19907043. 37. Jefferson T, y col. Multisystem failure: the story of anti-influenza drugs. BMJ. 2014;348:g2263. doi: 10.1136/bmj.g2263. PMID:24721793.

38. Abramson JD, y col. Should people at low risk of cardiovascular disease take a statin? BMJ. 2013;347:f6123. Erratum in: BMJ. 2014;348:g3329. doi: 10.1136/bmj.f6123. PMID:24149819. 39. Ioannidis JP, y col. Increasing value and reducing waste in research design, conduct, and analysis. Lancet. 2014;383(9912):166-75. doi: 10.1016/S0140-6736(13)62227-8. PMID:24411645.

40. Glasziou P. y col. Reducing waste from incomplete or unusable reports of biomedical research Lancet. 2014:383(9913):267-76. doi: 10.1016/S0140-6736(13)62228-X. PMID:24411647.

41. Chan AW, y col. Increasing value and reducing waste: addressing inaccessible research. Lancet. 2014;383(9913):257-66. doi: 10.1016/S0140-6736(13)62296-5. PMID:24411650.

42. loannidis JP, y col. Early or deferred zidovudine therapy in HIV-infected patients without an AIDS-defining illness. Ann Intern Med. 1995;122(11):856-66. PMID:7741372 doi: 10.7326/0003-4819-122-11-199506010-00009.

43. Jørgensen AW, y col. Cochrane reviews compared with industry supported meta-analyses and other meta-analyses of the same drugs: systematic review. BMJ. 2006;333(7572):782. PMID:17028106 doi: 10.1136/bmj.38973.444699.0b

44. Bell CM, y col. Bias in published cost effectiveness studies: systematic review. BMJ. 2006;332(7543):699-703. PMID:16495332 doi: 10.1136/bmj.38737.607558.80.

45. Lenzer J, y col. Ensuring the integrity of clinical practice guidelines: a tool for protecting patients. BMJ. 2013;347:f5535. doi: 10.1136/bmi.f5535. PMID:24046286.

46. Altman DG. ISIS and the emergence of large, simple trials. Lancet. 2015;386 (9994):636-7. doi: 10.1016/S0140-6736(15)61450-7. PMID:26334142.

47. Siontis KC, y col. Magnitude of effects in clinical trials published in high-impact general medical journals. Int J Epidemiol. 2011;40(5):1280-91. doi: 10.1093/jie/dyr095. PMID:22039194. 48. loannidis JP, y col. Estimates of the continuously publishing core in the scientific workforce. PLoS ONE. 2014;9(7):e101698. doi: 10.1371/journal.pone.0101698. eCollection 2014. PMID:25007173.

49. Emanuel EJ. Reinventing American health care. PublicAffairs, New York, 2014.

50. Fleurence R, y col. Launching PCORnet, a national patient-centered clinical research network. J Am Med Inform Assoc. 2014;21(4):578-82. doi: 10.1136/amiajnl-2014-002747.

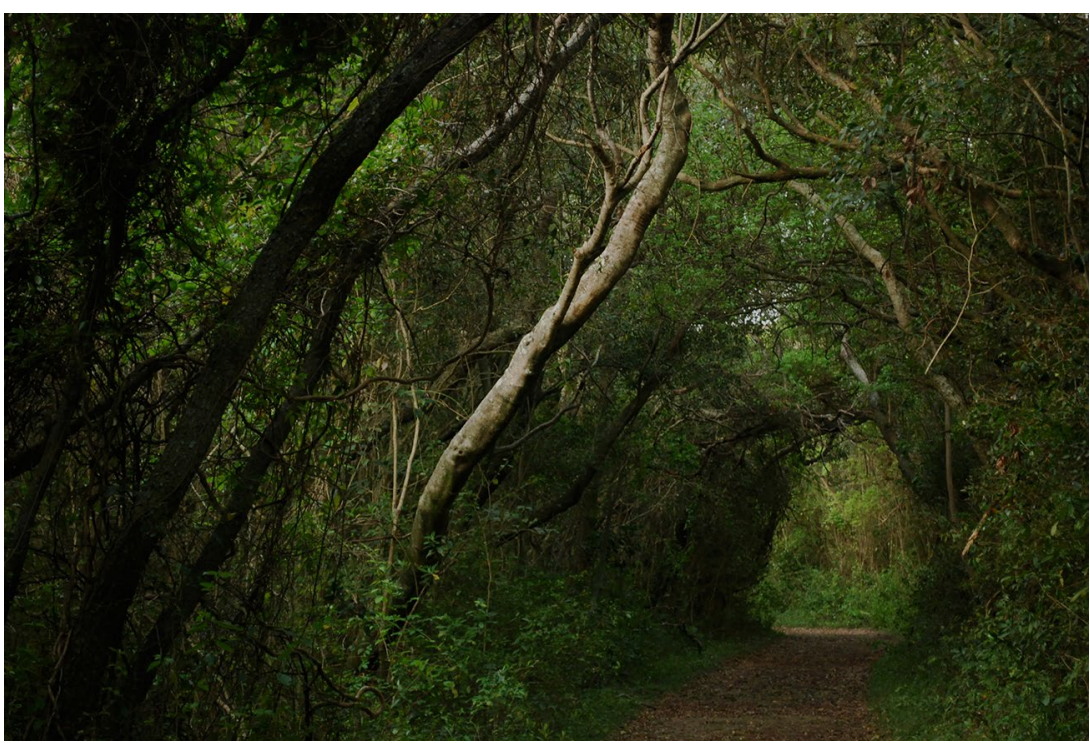

Cortesía de Silvia Spina 